\title{
A Novel Model for Individual Risk Quantification of Pressure Pipeline
}

\author{
Zhang Ying ${ }^{1}$, Zhao Xiaoxue ${ }^{1}$, Peng Lei ${ }^{2}$, Wang Wei1 ${ }^{1, *}$ and Dan Guo ${ }^{3}$ \\ ${ }^{1}$ School of Emergency Management, Henan Polytechnic University, Jiaozuo 454003, China \\ ${ }^{2}$ School of Energy Science and Engineering, Henan Polytechnic University, Jiaozuo 454003, China \\ ${ }^{3}$ Science Faculty, University College Cork, Cork 999014, Ireland
}

Received 02 December 2020; Accepted 22 August 2021

\begin{abstract}
Due to the high risk of pressure pipeline, the risk management of pressure pipeline is an important part of national public safety risk prevention. To quantify the individual risk of pressure pipeline, explain the interaction mechanism of each factor clearly, and identify the risk level more accurately, the risk factors of the risk subject and receptor were identified by using the Pressure-State-Response (PSR) model and the vulnerability model, the comprehensive evaluation mathematical model of the individual risk of the pressure pipeline was established using the factor analysis and mutational progression method, the model is applied to the risk level evaluation of the $185 \mathrm{~km}$ Jinjing pipeline of China Aviation Oil Group. Results show that the overall individual risk level of 61 evaluation units in Jinjing pipeline is low, and the individual risk values of 42 pipe sections are concentrated in [0.85,0.9). The individual risks of pipe sections 9 , $24,27,34,40,45,54$, and 55 are unacceptable and should be supervised. The conclusion obtained from this study provides a novel method for the government regulators to risk management of the pressure pipeline.
\end{abstract}

Keywords: Pressure pipeline, Individual risk mutation progression method, Quantitative mode

\section{Introduction}

With the sustained and rapid development of China's economy, the country's demand for energy will increase, especially the rapid development of eight high energyconsuming industries, such as metallurgy, power, building materials, and the chemical industry. The whole society's demand for pressure pipelines is also increasing. Pressure pipeline safety is gradually becoming an integral part of national public security [1]. In recent years, the pipeline is affected by many risk factors in the operation process, such as third-party damage, construction defects, and other issues, resulting in pipeline accidents. Given the high risk, pipeline accidents often bring serious casualties, large economic losses, and adverse social impacts.

Some of the major accidents cause disastrous consequences, such as mass casualties, large-scale transfer of residents, serious impact on large-scale production and life, destruction of traffic trunk lines, and large-scale environmental pollution [2]. The risk factors of the pressure pipeline itself (risk subject) bring greater individual risk to the surrounding residential, communities, and other public places (hereinafter referred to as the risk receptor). Therefore, in-depth study of pressure pipeline individual risk quantitative model and method and scientific and reasonable development of individual risk acceptable standard value become important issues to be solved.

\section{State of the Art}

2.1 Pressure pipeline risk evaluation technique

Risk assessment of pressure pipelines was carried out abroad

*E-mail address: wangwei@hpu.edu.cn

ISSN: 1791-2377 @ 2021 School of Science, IHU. All rights reserved.

doi:10.25103/jestr.144.23 for more than 30 years and its evaluation system has begun to take shape. Hence, developed to mature application stage and made certain achievements [3]. Many countries have established pipeline risk evaluation systems and effective evaluation techniques and methods for different types of pipelines, which were gradually transitioned from qualitative to quantitative analysis. Risk analysis has been gradually systematized and standardized and has entered a new stage of risk management [4]. The first pipeline risk analysis in 1985 was conducted in the United States. [5]. The Kent index quantitative risk assessment model introduced by Kent in the Pipeline Risk Management Manual is currently the most widely used and influential [6]. Since the early 1990s, Canada has started research on pipeline risk assessment and management techniques. In 1994, a professional pipeline risk assessment committee was set up to study the implementation of pipeline risk assessment technology development in the country. In addition, the first generation of pipeline risk assessment software was successfully developed by the famous Nova pipeline company in Canada [7]. In the UK, British Gas Group plc has also developed professional computer integrated manufacturing (CMI) risk software for risk assessment of urban gas pipelines [8]. In the pipeline risk management project research, the UK Health and Safety Executive Board (HSE) has also developed a MISHAP software package that can be used to calculate the pipeline failure probability and risk and obtain certain application results [9]. In the past 30 years of research and practice, a large number of foreign pressure pipelines have adopted the Kent method for risk assessment and analysis and developed risk assessment and management software suitable for domestic use. However, due to different specific conditions in different countries, most countries have improved to varying degrees according to specific national conditions on the basis of the Kent method, 
which resulted in a good application effect [10]. In recent years, foreign scholars have begun to try some quantitative risk assessment models. Senouci et al. established the failure model of pressure pipeline based on fuzzy logic using historical pipeline accident data [11]. Alzbutas et al. quantitatively evaluated the pipeline failure probability and gas combustion probability related to pipeline age by the Bayesian method using the historical data of gas pipelines [12]. Medeiros et al. established a multicriteria decisionmaking model for multidimensional risk assessment of pressure pipelines to provide a decision-making basis for pipeline maintenance [13]. Urbina et al. used the polynomial neural network method to optimize the evaluation model to deal with multivariate and uncertainty problems to improve the accuracy of risk assessment [14]. The research on risk assessment technology of pressure pipelines in China started relatively late compared with that in foreign countries. Since 1995, oil field enterprises and researchers have been studying the theory and application of pipeline risk assessment [15]. At present, many studies on qualitative and semiquantitative corrosion risk assessment of urban pipelines in China exist; where quantitative research is still in constant exploration. Based on foreign research results, domestic scholars use the Kent index, fault tree, BP neural network, Bayesian network model evaluation, principal component, and failure mode and effects analysis (FMEA) method to evaluate the risk of pressure pipeline failure. These methods have obtained good results to some extent, but limitations in the application of methods are still noticed [16]. At present, qualitative methods are widely used in practice, such as failure mode effects and criticality analysis (FMECA) method, fault tree analysis (FTA) method, liable equency consequence (LEC) evaluation method, etc. [17].

\subsection{Individual risk}

The methods for determining individual risk acceptance criteria mainly include the as low as reasonably practicable (ALARP) principle [18], risk matrix, annual fatality risk (AFR) value [19], and average individual risk (AIR) value [20-21]. Among them, the AFR value is widely used in different fields as a widely accepted method to calculate individual risk at home and abroad. AFR is the probability of a person dying within a year under certain conditions. The AFR method is more used because it is simple and has fast quantitative indicators of individual risk [22].

The determination of the acceptable level for individual risk is a complex subject. The research work in this field in China is late, and the research on the acceptable standard of individual risk is still in its infancy. The research focuses on the field of engineering safety. At present, some progress has been made in marine, nuclear power, construction, dam, petrochemical [23, 24], geological disasters [25], landslides [26], transportation [27], coal mines [28], and other industries and fields. Relevant studies discussed the level of acceptable risk in various industries in China from different angles. Gao and Liu analyzed the acceptable risks in the Netherlands, the UK, and other countries, as well as the definition method of acceptable criteria for individual risks and social risks. Therefore, the determination method of acceptable criteria for individual risks in the field of production safety in China was studied [22]. According to China's national conditions and the influencing factors of the dam risk standard, the suggestions of individual life risk and social risk standards are provided by Du et al. [29]. Zhao obtained the acceptable level of individual risk of natural geological disasters in China based on the analysis of geological disaster acceptable risk standard connotation and geological disaster risk estimation method [30]. Wang studied the influencing factors of individual risk perception and the construction of a theoretical model under sudden water pollution incidents, providing a reference for the optimization of individual behavior under sudden water pollution incidents [31]. Cheng built a probability model of pedestrian collision risk based on probability theory and calibrates pedestrian traffic delay and collision risk loss under different speed levels, hence providing a decisionmaking basis for the safety of urban road crossing pedestrians [32]. Zhang adopted the average personal risk value method to determine the reference range of individual acceptable risk standards of coal mines from 2013 to 2017 [28]. Li constructed the system dynamics model of the influencing factors of miners' risk perception from the individual perspective, simulates the dynamic evolution process of miners' risk perception level and the influence of various factors on each working period to explore the influencing factors of miners' risk perception in high-risk positions [33]. Wang used the system dynamics software Vensim to build a model to study the fitting process of the difference between the individual risk perception level and objective risk level, and then obtained several key time points and related measures for risk control [34]

\section{Methodology}

\subsection{Analysis framework of individual risk factors of pressure pipeline}

The individual risk of pressure pipeline includes two basic elements, namely, the possibility of pressure pipeline accidents and the severity of possible consequences. The possibility of a risk accident is affected by the inherent risk of the risk subject (namely risk), and the severity of the consequences is closely related to the inherent risk of the risk subject (namely risk) and the vulnerability of the risk receptor. Therefore, the individual risk of the pressure pipeline is determined in this study to include the risks of risk subjects and risk receptors.

\subsubsection{Identification of risk factors}

For the risk subject, the pressure-state-response (PSR) model is used to identify the influencing factors of the risk subject, where the pressure represents the factors that make the pipeline lose its stable state and the internal structure change; state represents the actual conditions and conditions of the pipeline in the process of operation; and responses indicate measures and countermeasures taken to eliminate pressure in order to restore the pipeline to a stable state. In comparison with the traditional risk identification method, the PSR model is more scientific, systematic, organized, and can also clearly explain the interaction mechanism of various elements in the risk subject.

The PSR model is a dynamic model, which solves the problem of why, under what conditions, and how to do it in the future. The PSR model is also suitable for the dynamic characteristics of individual risk of pressure pipelines. Therefore, this study uses the pressure, state, and response as the three elements to identify a specific scenario of risk subjects, including the following elements:

Pressure: Factors indicating the loss of stability and changes in the internal structure of the pipeline, including: 
(1) Actual working pressure ratio: The ratio of the maximum allowable operating pressure of the pipeline to the actual working pressure of the pipeline.

(2) Anticorrosion measures: Corrosion is the main form of pipeline failure.

(3) Minimum buried depth: The pressure pipe is generally laid underground and the minimum buried depth directly determines the soil geological conditions and the level of external influence on it.

(4) Occupying pressure: The Law of the People's Republic of China on Petroleum and Natural Gas Pipeline Protection stipulates those buildings and structures are prohibited within five meters of territory on both sides of the central line of the pipeline.

(5) Pipeline sign: The completeness of pipeline sign directly affects the safety of construction activities near the pipeline and indirectly affects the possibility of accidents.

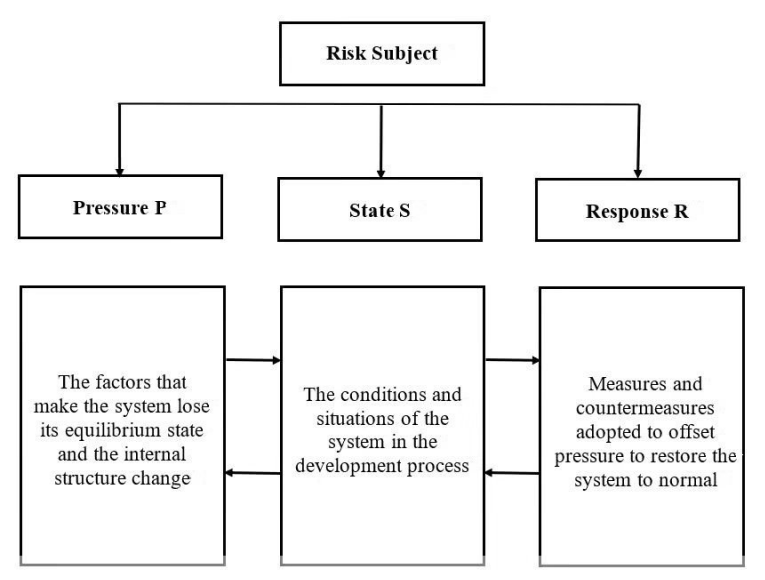

Fig.1. Pressure state response model

State: The actual conditions and conditions of the pressure pipeline during operation.

(1) Period factor: In certain special periods (holidays, rest days, nights, etc.), the number of staff in the post will decrease because of rest, resulting in inadequate personnel security measures relative to normal times.

(2) Geographical factors: The spatial position of the pipeline. Given the influence of disturbance factors, the possibility of accidents with sparse and dense personnel is different.

(3) Disaster factors: These factors mainly include rainstorms, floods, landslides, debris flow, earthquakes, etc. External interference is an important cause of failure accidents of pressure pipeline.

(4) Activity factors: These factors mainly refer to the activity of personnel near the pipeline. In general, the level of activity also determines the possibility of pipeline accidents to a certain extent.

Response: Measures and countermeasures adopted to eliminate pressure to restore the pipeline to a stable state.

(1) Line patrol frequency: Line patrol can detect the defects of the pipeline and the weak links that threaten the safe operation of the line in time.

(2) Pipeline monitoring system: The monitoring system can timely forecast data anomalies and other hidden dangers to control the development of hidden dangers.

(3) Configuration of safety management personnel: Personal quality and configuration of safety management personnel are closely related to the inherent risks of the pressure pipeline body.
(4) Support or construction of fire brigades: The strength of fire forces determines whether measures can be taken to kill them in the embryonic stage of the accident, affecting the possibility of pressure pipeline accidents.

The influencing factors of risk subjects are obtained, including the three first-level factors, namely, pressure, state, response, with 13 second-level factors.

\subsubsection{Identification of risk receptor factors}

Risk receptors in this study are characterized by vulnerability indices, which can be expressed as a function of sensitivity, exposure, and coping according to the definition of vulnerability. Sensitivity refers to the sensitivity of the system which is easy to damage in the face of interference due to its characteristics. Exposure refers to the system contact interference time, space, distance, etc. Coping ability refers to the system to deal with interference and to achieve the purpose of self-adjustment ability.

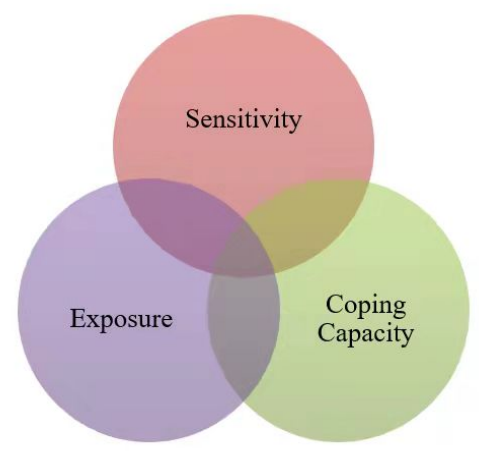

Fig.2. Risk receptor vulnerability model

\section{Sensitivity:}

(1) Age of personnel: Given the differences in age of personnel, significant differences in the instantaneous emergency response of personnel at the time of the accident exist.

(2) Personnel safety quality: Personnel with higher safety quality have stronger safety awareness, emergency responseability, and escape knowledge reserve than ordinary personnel, thus more likely to take corrective measures when accidents occur.

(3) Health status of personnel: Health status mainly refers to people's failure to receive and understand emergency evacuation information in time due to physical health reasons, thereby extending the evacuation time and reducing their evacuation capacity.

(4) The height of the facility: Affected by the number of building layers and when a high-rise building is in an accident, carrying out firefighting and rescue and evacuation considering the water supply capacity of the fire truck and its rescue height are relatively more difficult.

(5) Structural type of facility: Buildings also differ in impact resistance due to the use of different building materials and structures.

(6) Facility fire resistance: Facility fire resistance usually refers to the fire resistance grade, which is a grading scale to measure the fire resistance of a building. The fire resistance grade is mainly determined by the combustion performance and the minimum fire resistance limit of the building components.

(7) Functional importance of facilities: Some important infrastructures in the area around the pressure pipeline accident may be observed. Once the accident occurs, these important facilities are damaged, which not only affects the 
facility itself but also brings secondary disasters and aggravates the losses caused by the accident.

Exposure:

(1) Exposure time: In some special periods, the crowd gathered in public places is larger. If an accident occurs, the consequences of the accident are more serious.

(2) Exposure space: The spatial location of the risk receptor.

(3) Exposure distance (radius): The closer the location of the protective target is to the accident point, the greater the impact of accidental energy release will be, and the more serious the casualties and damage to buildings will be.

Coping ability:

(1) Information channels: Whether the government can release evacuation information timely and effectively, methods of releasing specific measures to the society to avoid or mitigate hazards, and whether the public can receive evacuation information in time and take corresponding actions will directly affect the surrounding casualties.

(2) The construction of emergency rescue plan: According to the Production safety accident emergency plan evaluation guide for production and business units, the preparation of an emergency plan should be legitimate, complete, scientific, targeted, practical, scientific, operational, and cohesive.

(3) Emergency drills: Emergency drills can enable emergency teams to accumulate experience in dealing with accidents and enhance the ability of overall collaboration and security capabilities.

(4) The allocation of emergency resources: The effective allocation of emergency resources determines if the deterioration of the situation can be curbed in time after the pressure pipeline accident. The accident consequences can be minimized.

The influencing factors of risk receptors are obtained, including the three first-level factors, namely, sensitivity, exposure, coping ability, and 14 second-level factors.

3.2 Individual risk factors screening of pressure pipeline After the preliminary formulation of the system of individual risk influencing factors of pressure pipelines, screening and optimizing the indicators are necessary. In this study, the factor analysis method is used to screen the primary indicators.

Factor analysis is the use of dimensionality reduction of mathematical ideas with a few potential variables (infectors) to describe the covariance relationship between most variables with a few comprehensive indicators to explain all the indicator information.

To measure whether the variables are suitable for factor analysis, the size of Kaiser-Meyer-Olkin (KMO) value and $P$-value of Bartlett's spherical test is determined. Kaiser believes that the higher the KMO value, the more common the variables are and the more suitable for factor analysis. In general, table 1 determines if factor analysis is possible.

Table 1. KMO index value judgment criteria

\begin{tabular}{c|c|c|c|c}
\hline $\mathrm{KMO}<0.5$ & $0.5<\mathrm{KMO}<0.7$ & $0.7<\mathrm{KMO}<0.8$ & $0.8<\mathrm{KMO}<0.9$ & $\mathrm{KMO}>0.9$ \\
\hline Misfit & General & Medium & Good & Excellent \\
\hline
\end{tabular}

When the KMO value is not suitable and can be determined according to the $P$-value. If the $P$-value is less than 0.05, the null hypothesis of Bartlett's test can be rejected and considered suitable for factor analysis.

According to the 27 elements identified by the above individual risk, a questionnaire is constructed. The questionnaire designed in this study consists of two parts. The first part is the basic information of the respondents, including gender, education level, and professional background. While the second part is the survey on the importance of indicators, including the measurement of risk subjects and risk receptors. Each plate is divided into three different levels according to the specific theoretical model. The questionnaire uses the Likert-5 scale to measure the items, which from 1 to 5 represents the degree of unimportant to very important. After index optimization by factor analysis, the individual risk evaluation model of the pressure pipeline is obtained. According to the requirements of the catastrophe model based on catastrophe theory for classification and hierarchical processing, this study constructs the pressure pipeline individual risk evaluation index system including '2 categories-22 indicators', as shown in Figure 3.

3.3 Quantitative model of individual risk of pressure pipeline based on mutation progression method

In the comprehensive evaluation of the individual risk of pressure pipelines, the establishment of an evaluation system and the determination of the weight of evaluation factors are the key issues in the study. In the current research at home and abroad, scholars often use the analytic hierarchy process, entropy method, grey clustering analysis, Delphi method, and fuzzy comprehensive evaluation method to determine the weight of the evaluation system. However, these methods often have greater subjectivity and uncertainty in determining the index weight.

In the context of the theoretical research of catastrophe theory, an application branch has emerged called the mutation progression method, which absorbs the advantages of the above common methods and does not need to consider the weight of each index in reducing subjectivity. The mutation series method only considers the relative importance of indicators, which has good applicability for the quantitative evaluation of the system and can solve the above problems encountered in the process of individual risk evaluation of pressure pipelines.

From the normalization formula, the control variables of different states in each layer of the system can be transformed into the states represented by the state variables. The elementary mutation model and normalization formula can be summarized to obtain Table 2 .

Although the mutation series method has something in common with the fuzzy subjection function, it has great advantages in practical application. In the catastrophe model, due to the characteristics of the model itself, the dominant role of control variables for the system state is determined by the model itself in the primary and secondary without the need for experts and scholars to give the weight. The evaluation methods are mostly determined by the subjective assignment method, and the results are often affected by the subjective conditions of the evaluator, thus lack of certain scientific and accuracy. 


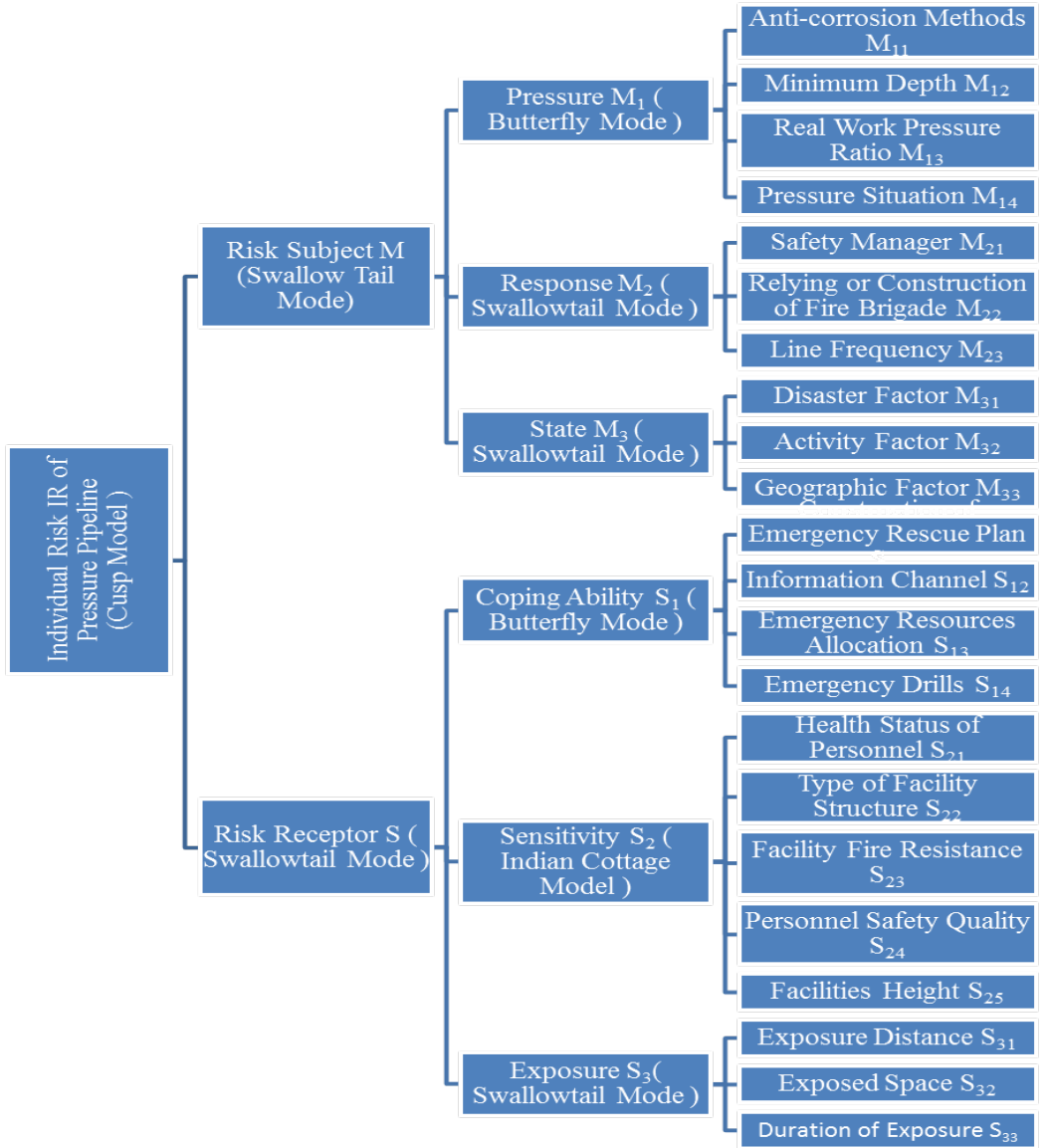

Fig.3. Individual risk assessment model of pressure pipeline based on catastrophe progression method

Table 2. Types of primary mutations and their normalization formulas

\begin{tabular}{c|c}
\hline Mutation type & Normalization formula \\
\hline Folded form & $x_{u}=\sqrt{|u|}$ \\
The Cusp & $x_{u}=\sqrt{|u|}, x_{v}=\sqrt[3]{|v|}$ \\
Swallow-tailed & $x_{u}=\sqrt{|u|}, x_{v}=\sqrt[3]{|v|}, x_{w}=\sqrt[4]{|w|}$ \\
Butterfly shape & $x_{u}=\sqrt{|u|}, x_{v}=\sqrt[3]{|v|}, x_{w}=\sqrt[4]{|w|}, x_{t}=\sqrt[5]{|t|}$ \\
Indian cottage type & $x_{u}=\sqrt{|u|}, x_{v}=\sqrt[3]{|v|}, x_{w}=\sqrt[4]{|w|}, x_{t}=\sqrt[5]{|t|}, x_{s}=\sqrt[6]{|S|}$ \\
\hline
\end{tabular}

\subsection{Determination of total catastrophe series}

According to the different number of control variables and the specific catastrophe model, the catastrophe level values of each layer index are calculated by the normalization formula, and the recursive calculation is carried out step by step upward. The total catastrophe-level of individual risk of pressure pipeline can be obtained.

In the process of step-by-step calculation, according to the steps of the mutation progression method, the indexes of each level need to determine noncomplementary and complementary principles:

The complementary principle is when the control variables of the system can make up for each other's deficiencies, that is, a significant correlation between the control variables and the average value of the catastrophe level values at all levels should be selected as the total catastrophe-level value. For example:

$$
I R=\frac{1}{2} M^{i}+S^{j},\{i, j\}=\left\{\frac{1}{2}, \frac{1}{3}\right\}
$$

Where $I R$ is the individual risk value of pressure pipeline and $M$ and $S$ represent the catastrophe level values of risk subject and risk receptor, respectively.

On the contrary, if the role of each control variable in a system cannot be replaced by each other, hence they cannot make up for each other's shortcomings. At this time, the minimum value of the catastrophe level value in each level must be selected as the total catastrophe-level value. For example:

$I R=\operatorname{in}\left\{M^{i}, \mathrm{~S}^{j}\right\},\{i, j\}=\left\{\frac{1}{2}, \frac{1}{3}\right\}$

According to the different structural types and internal relations of the individual risk evaluation index system of pressure pipeline, the interaction relationship of each level index is obtained, as shown in Table 3. 
Table 3. Selection of principles at all levels

\begin{tabular}{c|c} 
Individual risk level & Principle selection \\
\hline First floor $(M, S)$ & complementation \\
Second floor $\left(M_{1}, M_{2}, M_{3}\right)$ & complementation \\
Second floor $\left(S_{1}, S_{2}, S_{3}\right)$ & complementation \\
Third floor $\left(M_{11}, M_{12}, M_{13}, M_{14}\right)$ & non-complementary control \\
Third floor $\left(M_{21}, M_{22}, M_{23}\right)$ & complementation \\
Third floor $\left(M_{31}, M_{32}, M_{33}\right)$ & non-complementary control \\
Third floor $\left(S_{11}, S_{12}, S_{13}, S_{14}\right)$ & complementation \\
Third floor $\left(S_{21}, S_{22}, S_{23}, S_{24}, S_{25}\right)$ & complementation \\
Third floor $\left(S_{31}, S_{32}, S_{33}\right)$ & non-complementary control \\
\hline
\end{tabular}

\subsection{Risk subject evaluation model}

The evaluation models of pressure $\left(M_{1}\right)$, response $\left(M_{2}\right)$ and state $\left(M_{3}\right)$ are obtained as follow:

$$
\begin{aligned}
M_{1}= & \min \left\{M_{11}{ }^{i}, M_{12}{ }^{j}, M_{13}{ }^{k}, M_{14}{ }^{p}\right\},\{i, j, k, p\} \\
& =\left\{\frac{1}{2}, \frac{1}{3}, \frac{1}{4}, \frac{1}{5}\right\} \\
M_{2} & =\frac{1}{3} M_{21}{ }^{i}+M_{22}{ }^{j}+M_{23}{ }^{k},\{i, j, k\}=\left\{\frac{1}{2}, \frac{1}{3}, \frac{1}{4}\right\} \\
M_{3} & =\min \left\{M_{31}{ }^{i}, M_{32}{ }^{j}, M_{33}{ }^{k}\right\},\{i, j, k\}=\left\{\frac{1}{2}, \frac{1}{3}, \frac{1}{4}\right\}
\end{aligned}
$$

Therefore, the evaluation model of risk subjects can be obtained as follows:

$I R=\frac{1}{2} M^{i}+S^{j},\{i, j\}=\left\{\frac{1}{2}, \frac{1}{3}\right\}$

\section{Where}

$M$ is the catastrophe value of risk subject;

$M_{1}$ is the catastrophe level value of the secondary index 'pressure' of the risk subject;

$M_{11}, M_{12}, M_{13}$, and $M_{14}$ are the dimensionless raw data of the three-level indicators of pressure;

$M_{2}$ is the catastrophe level value of the secondary index response of the risk subject;

$M_{21}, M_{22}$, and $M_{23}$ are the dimensionless raw data of the three-level indicators of response, respectively;

$M_{3}$ is the catastrophe level value of the secondary index state of the risk subject; and

$M_{31}, M_{32}$, and $M_{33}$ are the dimensionless original data of the three-level indicators of state, respectively.

\section{(1) Risk receptor evaluation model}

The evaluation models of coping ability $\left(S_{1}\right)$, sensitivity $\left(S_{2}\right)$, and exposure $\left(S_{3}\right)$ are obtained as follow:

$$
\begin{aligned}
S_{1}= & \frac{1}{4}\left(S_{11}{ }^{i}+S_{12}{ }^{j}+S_{13}{ }^{k}+S_{14}{ }^{p}\right),\{i, j, k, p\} \\
& =\left\{\frac{1}{2}, \frac{1}{3}, \frac{1}{4}, \frac{1}{5}\right\} \\
S_{2}= & \frac{1}{5} S_{21}{ }^{i}+S_{22}{ }^{j}+S_{23}{ }^{k}+S_{24}{ }^{p}+S_{25}{ }^{q},\{i, j, k, p, q\} \\
& =\left\{\frac{1}{2}, \frac{1}{3}, \frac{1}{4}, \frac{1}{5}, \frac{1}{6}\right\}
\end{aligned}
$$

$S_{3}=\min \left\{S_{31}{ }^{i}, S_{32}{ }^{j}, S_{33}{ }^{k}\right\},\{i, j, k\}=\left\{\frac{1}{2}, \frac{1}{3}, \frac{1}{4}\right\}$

Therefore, the evaluation model of the risk receptor can be obtained as follows:

$$
S=\frac{1}{3} S_{1}^{i}+S_{2}^{j}+S_{3}^{k},\{i, j, k\}=\left\{\frac{1}{2}, \frac{1}{3}, \frac{1}{4}\right\}
$$

Where

$S$ is the mutation value of the risk receptor;

$S_{1}$ is the mutation level value of the secondary index of risk receptor's coping ability;

$S_{11}, S_{12}, S_{13}$, and $S_{14}$ are the dimensionless original data of the three-level indicators of coping ability;

$S_{2}$ is the mutation level value of the second-order index sensitivity of the risk receptor;

$S_{21}, S_{22}, S_{23}, S_{24}$, and $S_{25}$ are the dimensionless original data of three-level indicators of response respectively;

$S_{3}$ is the mutation value of the second-order indicator exposure of the risk receptor; and

$S_{31}, S_{32}$, and $S_{33}$ are the dimensionless original data of the three-level indicators of exposure;

\section{(2) Individual risk comprehensive evaluation model of pressure pipeline}

From the evaluation model of risk subject and risk receptor, the comprehensive evaluation model of the individual risk of pressure pipeline based on mutation progression method can be obtained:

$I R=\frac{1}{2} M^{i}+S^{j},\{i, j\}=\left\{\frac{1}{2}, \frac{1}{3}\right\}$

Where

$I R$ is the comprehensive evaluation value of the individual risk of pressure pipeline;

$M$ is the catastrophe value of risk subject; and

$S$ is the mutation level of the risk receptor.

\section{Results Analysis and Discussion}

\subsection{Individual risk assessment of China Civil Aviation Jinjing Pipeline}

The total length of China Civil Aviation Jinjing Pipeline is 185 kilometers with two municipalities and six administrative regions. Among them, Tianjin section with 133 km (Tanggu District, Dongli District, Beichen District, and Wuqing District), Beijing section with $52 \mathrm{~km}$ (Tongzhou 
District, Shunyi District), through the Haihe River, the North Canal, Xinkai River, Yongding New River, and other 12 large and medium-sized rivers through 52 small rivers, 12 railroads, and 43 highways. A total of 12 valve chambers exists along the line, including nine in Tianjin section (four in Tanggu District, two in Beichen District, and three in Wuqing District), and three in Tongzhou District of Beijing section. At present, the annual maximum transport capacity of 275 million tons accounts for more than $75 \%$ of the capital airport oil.

According to the PSR model of risk subjects, the data of the 61 sections in the Beijing-Tianjin Pipeline on a working day in March are collected. After the completion of data information collection, the underlying indicators are dimensionless. According to the above formula, the evaluation values of risk subjects, risk receptors, and individual risks are as follow:

Table 4. Selection of principles at all levels

\begin{tabular}{|c|c|c|c|c|c|c|c|}
\hline Number & Individual Risk & Risk Subject & Risk Receptor & Number & Individual Risk & Risk Subject & Risk Receptor \\
\hline 1 & 0.89 & 0.68 & 0.87 & 32 & 0.85 & 0.66 & 0.69 \\
\hline 2 & 0.90 & 0.72 & 0.87 & 33 & 0.88 & 0.77 & 0.70 \\
\hline 3 & 0.91 & 0.75 & 0.87 & 34 & 0.78 & 0.45 & 0.70 \\
\hline 4 & 0.94 & 0.84 & 0.87 & 35 & 0.86 & 0.69 & 0.70 \\
\hline 5 & 0.93 & 0.83 & 0.87 & 36 & 0.86 & 0.69 & 0.70 \\
\hline 6 & 0.93 & 0.83 & 0.88 & 37 & 0.86 & 0.78 & 0.59 \\
\hline 7 & 0.85 & 0.55 & 0.88 & 38 & 0.85 & 0.76 & 0.57 \\
\hline 8 & 0.85 & 0.56 & 0.88 & 39 & 0.85 & 0.74 & 0.57 \\
\hline 9 & 0.81 & 0.45 & 0.88 & 40 & 0.76 & 0.49 & 0.57 \\
\hline 10 & 0.86 & 0.59 & 0.85 & 41 & 0.83 & 0.71 & 0.57 \\
\hline 11 & 0.91 & 0.76 & 0.85 & 42 & 0.83 & 0.67 & 0.62 \\
\hline 12 & 0.92 & 0.80 & 0.86 & 43 & 0.84 & 0.67 & 0.63 \\
\hline 13 & 0.93 & 0.83 & 0.86 & 44 & 0.86 & 0.72 & 0.64 \\
\hline 14 & 0.93 & 0.83 & 0.86 & 45 & 0.79 & 0.51 & 0.66 \\
\hline 15 & 0.90 & 0.74 & 0.85 & 46 & 0.87 & 0.75 & 0.66 \\
\hline 16 & 0.82 & 0.48 & 0.86 & 47 & 0.90 & 0.73 & 0.85 \\
\hline 17 & 0.82 & 0.48 & 0.85 & 48 & 0.83 & 0.49 & 0.87 \\
\hline 18 & 0.82 & 0.47 & 0.85 & 49 & 0.83 & 0.49 & 0.87 \\
\hline 19 & 0.89 & 0.71 & 0.85 & 50 & 0.83 & 0.49 & 0.87 \\
\hline 20 & 0.90 & 0.74 & 0.82 & 51 & 0.85 & 0.55 & 0.88 \\
\hline 21 & 0.89 & 0.70 & 0.81 & 52 & 0.83 & 0.49 & 0.86 \\
\hline 22 & 0.86 & 0.70 & 0.71 & 53 & 0.89 & 0.79 & 0.73 \\
\hline 23 & 0.80 & 0.46 & 0.78 & 54 & 0.80 & 0.49 & 0.73 \\
\hline 24 & 0.78 & 0.46 & 0.70 & 55 & 0.80 & 0.49 & 0.73 \\
\hline 25 & 0.85 & 0.66 & 0.70 & 56 & 0.91 & 0.84 & 0.73 \\
\hline 26 & 0.86 & 0.70 & 0.70 & 57 & 0.88 & 0.73 & 0.73 \\
\hline 27 & 0.77 & 0.42 & 0.69 & 58 & 0.92 & 0.84 & 0.76 \\
\hline 28 & 0.85 & 0.67 & 0.71 & 59 & 0.92 & 0.84 & 0.76 \\
\hline 29 & 0.85 & 0.67 & 0.71 & 60 & 0.93 & 0.84 & 0.85 \\
\hline 30 & 0.86 & 0.70 & 0.71 & 61 & 0.93 & 0.84 & 0.85 \\
\hline 31 & 0.86 & 0.70 & 0.69 & & & & \\
\hline
\end{tabular}

Through the above calculation, the individual risk value of the pressure pipeline can be determined. The distribution of the individual risk value of 61 segments is analyzed with 0.05 as the gradient, as shown in Figure 4.

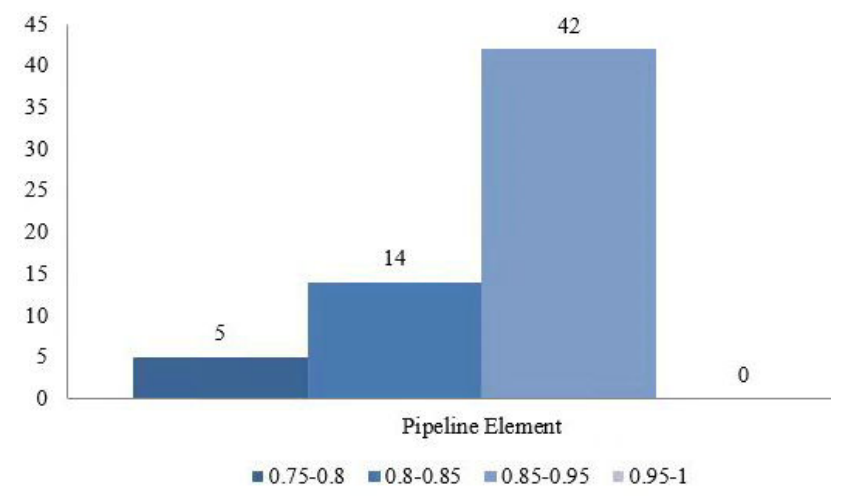

Fig.4. Distribution of individual risk values for 61 segments

A total of 61 evaluation units have low overall individual risk levels and good security situations. The individual risk values of 42 segments are concentrated in (0.85 and 0.9) accounting for $68.85 \%$, fourteen units in $(0.8$ and 0.85$)$ accounting for $22.95 \%$; five evaluation units in $(0.75$ and 0.8 ), accounting for $8.2 \%$; and the individual risk value without evaluation unit is $(0.95$ and 1$)$.
Most of the pipe sections are concentrated in [0.8, 0.9], and no pipe section in $[0.95,1)$, indicating that the overall safety of the Jinjing pipeline is good. Enterprises can adopt scientific and effective safety management methods, timely investigate security risks, and do a good job in the prevention of various security issues. However, due to the lack of experience in dealing with unexpected events in oil pipeline transportation, some deficiencies in the construction of emergency plans, emergency drills, and the allocation of emergency resources are still observed.

\subsection{Determination of acceptable criteria}

According to the distribution characteristics of individual risk value of Jinjing pipeline and the principle of Hugh hart control chart, the acceptance criterion of individual risk of pressure pipeline is set. For the data subject to the normal distribution, the Hugh hart control chart can be used for discrimination. First, the data of 61 units are tested for normal distribution, with skewness and kurtosis at 0.159 and 0.671 , respectively. The standard error does not reach 0.05 significant levels. Therefore, this group of data approximately presents normal distribution. In the figure, a 45-degree diagonal as the theoretical normal cumulative probability distribution line and another actual cumulative probability distribution line composed of small circles can be noticed. Through the comparison of the two, the data in 
this example are distributed near the straight line, and approximately present a normal distribution

After testing, the data of 61 segments conform to the normal distribution and the Hugh hart control chart can be used to determine the grading standard of individual risk.
If $\sigma$ is the mean square deviation, $\mu$ is the mean value of the sample, and the control line $\mu \pm \sigma$ is taken, where $68.27 \%$ of the samples can be controlled, which meets the Pareto principle. Therefore, the set pipeline individual risk classification standard is as follows:

Table 5. Pipeline individual risk classification standards

\begin{tabular}{c|c|c|c|c}
\hline Individual Risk level & I (High Risk) & II (Medium Risk) & III (Low Risk) & IV (Low Risk) \\
\hline IR & $(0,0.82)$ & {$[0.85 .0 .86]$} & {$[0.86,0.91)$} & {$[0.91,1)$} \\
\hline
\end{tabular}

According to the ALARP principle, risks are divided into intolerance areas, ALARP areas (minimum reasonable feasible areas), and acceptable areas, as shown in Fig 5.

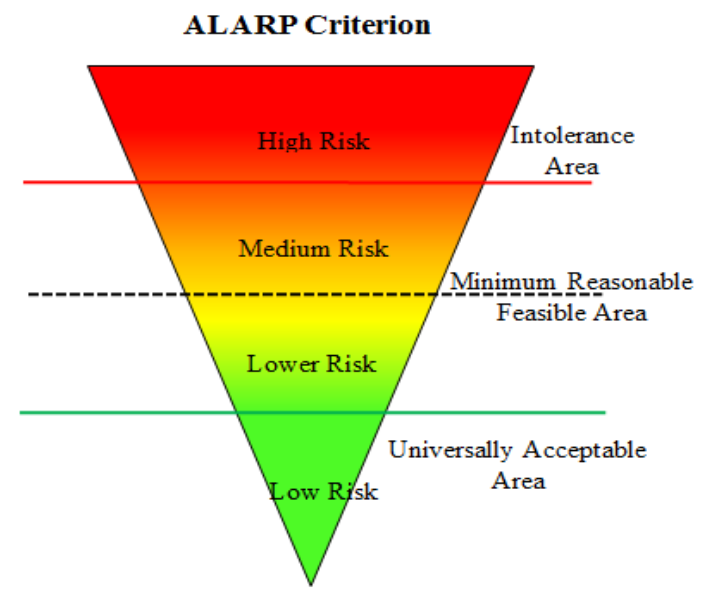

Fig 6 shows that the red line is the control line of acceptable risk. When the value of individual risk is lower than the red line, individual risk is an unacceptable risk. Therefore, the individual risks of pipes 9, 24, 27, 34, 44, 45, 54 , and 55 are unacceptable and should be monitored. Enterprises should formulate a sound and targeted safety inspection plan, implement comprehensive and focused management of weak links with low scores, and increase investment in safety manpower, material resources, and funds. Enterprises should make different safety investments according to different risk levels of segment units. When the segment is at high risk, the proportion of safety investment should be increased. When the segment is at low risk, the proportion of safety investment should be reduced accordingly, and the regulatory resources should be allocated more efficiently and reasonably to achieve the level of scientific, systematic, and effective safety management.

Fig.5. ALARP criterion diagram

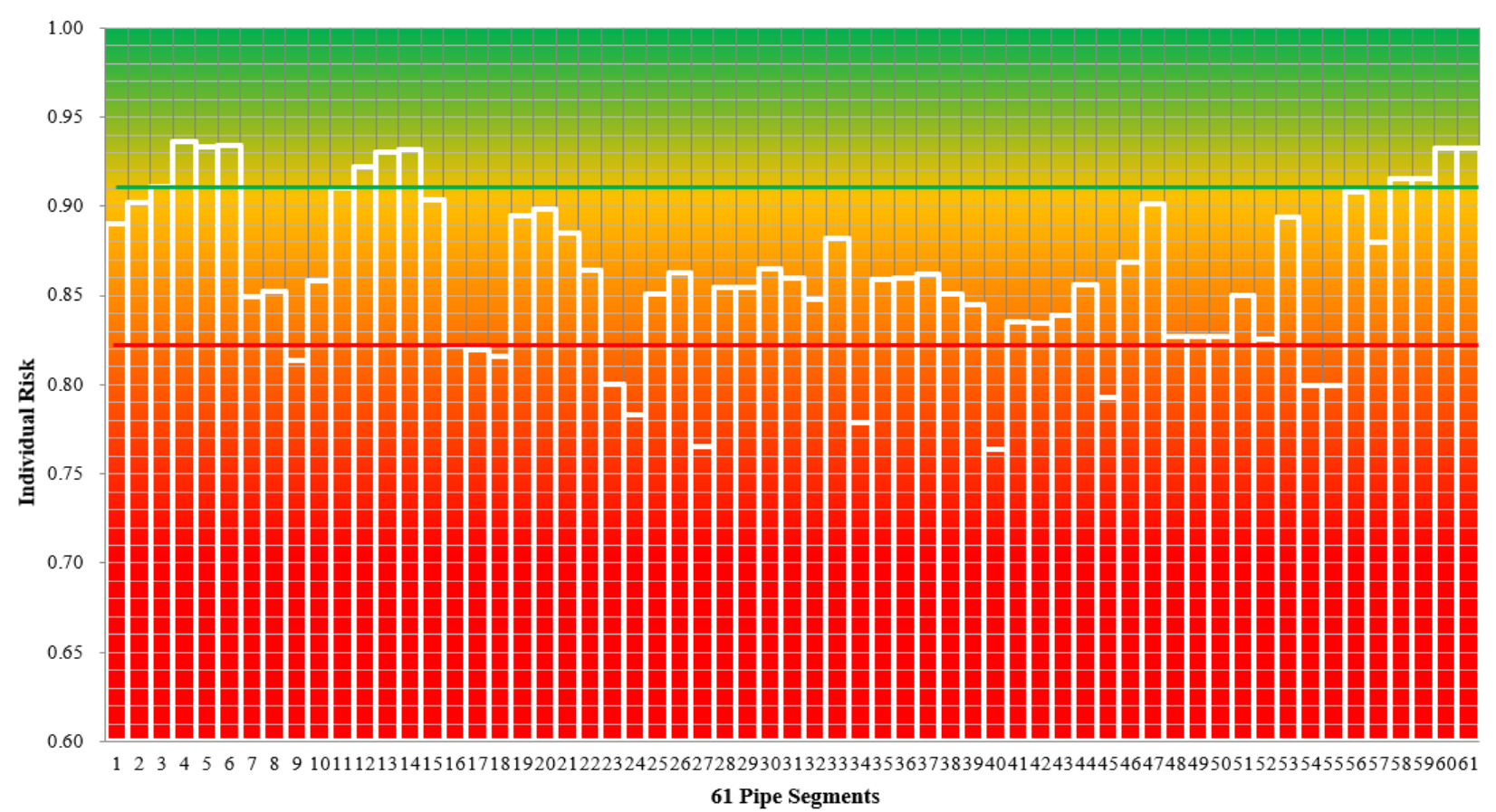

Fig.6. 61 individual risk acceptance criteria

61 Pipe Segments

The ALARP area between the red line and the green line should determine whether to take measures to reduce risk according to the specific circumstances; if it falls below the lower limit of ALARP, it is the risk negligible area, and its risk can be ignored.

\section{Conclusion}

The mutation progression method used in this study is a comprehensive evaluation method based on catastrophe theory and fuzzy mathematics theory for ranking analysis of evaluation objectives. The advantage of this method is to determine the relative importance of each index without weighting each index in reducing the subjectivity, ensuring the scientific and rationality, and the calculation is accurate and its application range is wide. Applying the catastrophe progression method to individual risk analysis of pressure pipelines can reduce the subjectivity of evaluation. In this study, the model of individual risk identification analysis of pressure pipeline was constructed and the quantitative 
evaluation model of individual risk of pressure pipeline based on catastrophe progression method was constructed, which provides a practical, effective, and scientific method for the safety supervision of pressure pipeline. The following conclusions are obtained:

(1) Based on the detection and collection of the accident information of the pressure pipeline, the factors of the individual risk of the pressure pipeline are identified and analyzed. The formation mechanism of the individual risk of the pressure pipeline is analyzed and studied. The factors of the risk subject are identified through the PSR model. The factors of the risk receptor are identified through the sensitivity-exposure-response model, and the meaning of each factor is explained.

(2) In consideration of the risk subject and the risk receptor of the pressure pipeline, the factor analysis method is used to screen and optimize the factors of the risk subject and the risk receptor, and the individual risk model of the pressure pipeline is suitable for the catastrophe progression method is obtained. The mathematical model of individual risk comprehensive evaluation of pressure pipeline based on catastrophe progression method is established, and the acceptance criterion of individual risk of pressure pipeline is studied.
(3) The empirical analysis of the $185 \mathrm{~km}$ Jinjing pipeline of China Aviation Oil Group is carried out, and 61 evaluation units are divided. The evaluation results of the risk subjects, risk receptors, and individual risks of Jinjing pipeline are obtained, and the acceptance criteria and control measures are given.

If conditions permit, empirical research on time and regional series analyses can be carried out for different types of pressure pipelines to better optimize the quantitative evaluation model of individual risk of pressure pipelines. In addition, the acceptance criteria for individual risk of pressure pipelines need to be updated and improved in realtime.

\section{Acknowledgments}

This study was supported by the Science and Technology Planning Project of Henan Province (212102310384) and Ph.D. Fund of Henan Polytechnic University (B2017-62).

This is an Open Access article distributed under the terms of the Creative Commons Attribution License.

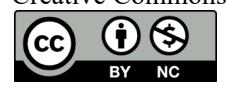

\section{References}

1. Alcaraz, M., Campos, R. A. "Framework based on Matlab/Simulink for the simulation of des using petri net models". International Journal of Simulation Modelling, 18(3), 2019, pp. 420-431.

2. Agudelo, M. A. L., Jóhannsdóttir, L., Davídsdóttir, B. “A literature review of the history and evolution of corporate social responsibility". International Journal of Corporate Social Responsibility, 4, 2019, pp. 1

3. Sabbaghi, M. M. "A supplier selection model emphasizing the project risk management in drug production in pharmaceutical industry". Tehnički Glasnik, 14(2), 2020, pp. 111-120.

4. Zhao, Y. T. "Risk assessment of oil-gas pipeline". Safety, (8), 2007, pp. 7-10.

5. Witter, R. E. "Guidelines for hazard evaluation procedures". Process Safety Progress, 11(2), 1992, pp. 50-52.

6. Han, Z. Y., Weng, W. G.. "Comparison study on qualitative and quantitative risk assessment methods for urban natural gas pipeline network". Journal of Hazardous Materials, 189(1-2), 2011, pp. 509-518.

7. Yacov, Y. H. "Total risk management". Risk Analysis, 11(2), 1991, pp. 169-171.

8. Jim, W. "The risk management process". Ergonomics Society of Australia, 21(3), 1992, pp. 16-19.

9. Coulson, K. E. W. "Pipe corrosion-conclusion: New guidelines promise more accurate damage assessment”. Oil Gas, 88(15), 1990, pp. 41-44.

10. Zorrilla, M. V., Petz, M., Agullo, T. M. S. "Ergonomic risk factors analysis with multi-hodological approach: Assessing workers' activities in buildings under construction". Dyna, 94(3), 2019, pp. 338-344.

11. Senouci, A., Elabbasy, M., Elwakil, E., Abdrabou, B., Zayed, T. “A Model for predicting failure of oil pipelines". Structure and Infrastructure Engineering, 10(3), 2014, pp. 375-387.

12. Alzbutas, R., Iešmantas, T., Povilaitis, M., Vitkute, J. "Risk and uncertainty analysis of gas pipeline failure and gas combustion consequence". Stochastic Environmental Research \& Risk Assessment, 28(6), 2014, pp. 1431-1446.

13. Medeiros, C. P., Alencar, M. H., De Almeida, A. T. "Multidimensional risk evaluation of natural gas pipelines based on a multicriteria decision model using visualization tools and statistical tests for global sensitivity analysis". Reliability Engineering \& System Safety, 165, 2017, pp. 268-276.

14. Urbinaa, G., Aoyama, A., Choi, E., "A polynomial neural network approach for improvement risk assessment and industrial safety". Icic Express Letters, 12(2), 2018, pp.97-107.
15. Świerzewski, M., Kalina, J. "Implementation of heat storage and network water cooler for improvement of energy and economic performance of municipal heating plant with biomass fired cogeneration module". Journal of Power Technologies, 99(2), 2019, pp. 131-141.

16. Ba, Z. N., Han, Y. X., Liang, J. W. "Risk assessment of the gas pipeline corrosion based on the improved AHP and fuzzy comprehensive evaluation method". Journal of Safety and Environment, 18(6), 2018, pp. 2103-2109.

17. Zhang, H. B., Feng, Q. S., Zheng, H. L. "Quantitative risk analysis of oil and gas long-distance pipeline". Chinese Journal of Safety Science, 18(3), 2008, pp. 161-165.

18. Nadim, F., Gudmestad, O. T. "Reliability of an engineering system under a strong earthquake with application to offshore platforms". Structure Safety, 14, 1994, pp. 203-217.

19. Montewka, J., Goerlandt, F., Kujala, P. "On a systematic perspective on risk for formal safety assessment (FSA)". Reliability Engineering \& System Safety, 127, 2014, pp. 77-85.

20. Vinnem, J. E. "Risk acceptance criteria for temporary phases". Journal of Offshore Mechanics and Arctic Engineering, 118, 1996, pp. 204-209.

21. Nyberg, L., Evers, M., Dahlström, M., Pettersson, A. "Sustainability aspects of water regulation and flood risk reduction in Lake Vnern". Aquatic Ecosystem Health \& Management, 17(4), 2014, pp. 331-340.

22. Gao, J. M., Wang, X. K., Zeng, M. R. "The research development and indication of the acceptable standard of individual risk and social risk". China's Production Safety Science and Technology, 6(3), 2007, pp. 29-34.

23. Zhao, X. W., Zhang, H., Luo, J. H. "Risk acceptance criteria for oil and gas pipelines". Oil and Gas Storage and Transportation, 35(1), 2016, pp. 1-6.

24. Zhang, Y., Qian, C. J., Zhang, M. G. "Study on risk perception quantitative calculation method for accidents in chemical industrial parks". Journal of Safety Science, 24(6), 2014, pp. 109-115.

25. Feng, D. M., Ning, L. J. "Factors of public risk perception of geological hazards in mining areas: Take Fushun west opencast mining area as an example". Science and Technology Promote Development, 16(8), 2020, pp. 901-908.

26. Lin, X. L., Guo, L. H. "Comparative study on acceptability of disasters in debris flow-prone areas: A case study of Dongchuan, Yunnan and Zhouqu, Gansu". Geosciences, 39(1), 2019, pp. 16-172.

27. Kochov, A., Danev, D., Kostikj, A. "Sustainable transport indicators in the context of introducing of electric passenger cars". Tehnick $k$ Glasnik, 14(3), 2020, pp. 318-324. 
28. Zhang, S., Li, X. L., Wu, C. "Study on acceptable risk criteria of coal industry in china". Journal of Safety Science, 25(1), 2015, pp. $158-164$

29. Du, X. W., Yang, J. "Discuss on risk standard of life loss caused by hydropower dam failure in China". Hydroelectric Power, (5), 2010, pp. 68-70.

30. Zhao, Z., Hou, E. K. "Study on life acceptable risk criteria of geological disasters in China". Science and Technology Report, 29(36), 2011, pp. 17-22.

31. Wang, M. X., Deng, X. M., Luo, X. Y. "Model establishment of the individual risk perception of water pollution emergencies". Safety and Environmental Engineering, 21(6), 2014, pp. 112-117.
32. Cheng, G. Z., Liu, B. T., Wu, L. X. "Urban road crossing pedestrian risk perception and decision loss". Transportation Systems Engineering and Information, 14(3), 2014, pp. 131-136.

33. Li, N. W., Wang, C. D., Niu, L. X., "Simulation of factors affecting individual risk perception of the high-risk position miner". Safety Production Science and Technology in China, 11(9), 2015, pp. 184190.

34. Wang, Z. Y., Zhang, C. Y. "System dynamics modeling and simulation of individual risk perception in chemical disaster" Science and Technology Management Research, 40(9), 2020, pp. 163-169. 